PROCEEDINGS OF THE

AMERICAN MATHEMATICAL SOCIETY

Volume 135, Number 12, December 2007, Pages 3771-3781

S 0002-9939(07)08911-3

Article electronically published on August 30, 2007

\title{
ALL TILTING MODULES ARE OF FINITE TYPE
}

\author{
SILVANA BAZZONI AND JAN ŠŤOVÍČEK
}

(Communicated by Martin Lorenz)

\begin{abstract}
We prove that any infinitely generated tilting module is of finite type, namely that its associated tilting class is the Ext-orthogonal of a set of modules possessing a projective resolution consisting of finitely generated projective modules.
\end{abstract}

\section{INTRODUCTION}

In the early eighties Brenner and Butler in [9] and Happel and Ringel in [18, generalized the classical Morita equivalence induced by a progenerator by introducing the notion of a tilting module over a finite-dimensional Artin algebra. In order to obtain equivalences between subcategories of the module category, tilting modules were assumed to be finitely generated and moreover of projective dimension at most one. Later, Miyashita 20 considered tilting modules of finite projective dimension and studied the equivalences induced by them. Colby and Fuller in 10 extended the setting to arbitrary rings, but in all the above-mentioned papers the tilting modules were always assumed to be finitely generated. The notion of infinitely generated tilting modules over arbitrary rings was introduced by Colpi and Trlifaj in [11] for the one-dimensional case and by Angeleri-Hügel and Coelho in [1] in the general case of finite projective dimension.

One of the advantages in dealing with infinitely generated tilting modules is evident in connection with the good approximation theory that they induce and which we are going to illustrate (cf. 3] and [1]). We recall that the tilting class $\mathcal{B}$ associated to a tilting module $T$ over a ring $R$ is the class of $R$-modules which are in the kernel of all the functors $\operatorname{Ext}_{R}^{i}(T,-)$. Such intersection of kernels is also called a right orthogonal of $T$ and denoted by $T^{\perp}$. If $\mathcal{A}$ denotes the class of the $R$-modules which are in the kernel of all the functors $\operatorname{Ext}_{R}^{i}(-, B)$, for any $B \in \mathcal{B}$, then the pair $(\mathcal{A}, \mathcal{B})$ of classes of $R$-modules is a hereditary cotorsion pair which induces special precovers and preenvelopes (cf. [16] and 24]). Alternatively, in the terminology used in [4] and [5] for classes of finitely generated modules, it induces right and left approximations.

Received by the editors October 1, 2005 and, in revised form, September 9, 2006.

2000 Mathematics Subject Classification. Primary 16D90, 16D30; Secondary 03E75, 16G99.

Key words and phrases. Tilting modules, cotorsion pairs.

The first author was supported by Università di Padova (Progetto di Ateneo CDPA048343 "Decomposition and tilting theory in modules, derived and cluster categories").

The second author was supported by a grant of the Industrie Club Duesseldorf, GAČR 201/05/H005, and the research project MSM 0021620839.

(C)2007 American Mathematical Society Reverts to public domain 28 years from publication 
There remained the problem of illustrating to what extent finitely and infinitely generated tilting modules are related. More precisely, the question was to decide whether the tilting classes are determined by finitely presented data in the sense that they are the right Ext-orthogonals of a set of finitely generated modules. There have been various efforts by many authors in order to solve this problem. A first answer was given by Eklof, Trlifaj and the first author in 7]. Using settheoretic methods, they show that one-dimensional tilting modules are of countable type in the sense that their associated tilting classes are the right Ext-orthogonal of a set of countably generated modules. Moreover, in [7] it is shown that the problem of determining whether a one-dimensional tilting module is of finite type is equivalent to proving that the associated tilting class is a definable class (see Section 2 for precise definitions). Herbera and the first author in [8] proved that every tilting module of projective dimension one is of finite type. The solution of the problem is obtained by proving that suitable countable inverse limits of groups of homomorphisms satisfy the Mittag-Leffler condition and that this condition is inherited by pure submodules. Almost at the same time Trlifaj and the second author in 22] were able to extend the set-theoretic methods used in [7] to the case of tilting modules of any finite-projective dimension and to prove that they are of countable type.

In the present paper, we prove that tilting modules of any finite projective dimension are of finite type. Let $\mathcal{S}$ be a set of modules possesing a projective resolution with finitely generated projective modules and such that all modules in $\mathcal{S}$ have projective dimension $\leq n$ for some natural number $n$. It is well known that the right Ext-orthogonal of such $\mathcal{S}$ is a tilting class (it follows by [1, Theorem 4.1] and [15, Theorem 2]). Our result thus states that every tilting class arises in this way. We call two tilting modules $T_{1}$ and $T_{2}$ equivalent if their corresponding classes coincide. As a consequence, every tilting module $T$ is up to equivalence determined by a set $\mathcal{S}$ of modules with projective resolutions formed by finitely generated projective modules as above. We then have $\mathcal{S}^{\perp}=T^{\perp}$, and in principle we can construct the tilting module $T$ from $\mathcal{S}$ using [15, Theorem 2].

First of all we note that the reduction to the countable case as proved in 22 is crucial. The next step is to prove that a tilting class is of finite type if and only if it is definable. In our situation this amounts to proving that, if $(\mathcal{A}, \mathcal{B})$ is the cotorsion pair induced by a tilting module, then every countably presented module in the class $\mathcal{A}$ is a direct limit of finitely presented modules in the class $\mathcal{A}$. This result will be achieved in Section 3 by using a particular notion of "freeness" for modules whose origin (for general algebraic structures) goes back to ideas introduced by Shelah to prove the singular compactness theorem. We say that a module is "free" if it admits a filtration of submodules with finitely presented factors. An adaptation of the proof of [14, XII.1.14] to our situation allows us to represent every countably presented module in the class $\mathcal{A}$ as a countable direct limit of finitely presented modules in the class $\mathcal{A}$. Our final result, Theorem 4.2, is obtained by induction on the projective dimension of a tilting module: we induct on the tilting cotorsion pairs induced by the syzygies of a tilting module and then we use arguments similar to the ones developed in [8] for the one-dimensional case. 


\section{Preliminaries}

In what follows, $R$ is always an associative ring with unit and all modules are right $R$-modules. First, we fix the terminology and recall some definitions.

$\mathcal{P}_{n}$ will denote the class of all modules of projective dimension at most $n$.

For a class $\mathcal{C}$ of modules and an infinite cardinal $\kappa$, we define $\mathcal{C}^{<\kappa}$ to be the class of all modules in $\mathcal{C}$ possessing a projective resolution consisting of $<\kappa$ generated projective modules.

An ascending chain $\left(M_{\alpha} \mid \alpha<\kappa\right)$ of submodules of a module $M$ indexed by a cardinal $\kappa$ is called continuous if $M_{\alpha}=\bigcup_{\beta<\alpha} M_{\beta}$ for all limit ordinals $\alpha<\kappa$. It is called a filtration of $M$ if $M_{0}=0$ and $M=\bigcup_{\alpha<\kappa} M_{\alpha}$.

Given a class $\mathcal{C}$ of modules, we say that a module $M$ is $\mathcal{C}$-filtered if it admits a filtration $\left(M_{\alpha} \mid \alpha<\kappa\right)$ such that $M_{\alpha+1} / M_{\alpha}$ is isomorphic to some module in $\mathcal{C}$ for every $\alpha<\kappa$. In this case we say that $\left(M_{\alpha} \mid \alpha<\kappa\right)$ is a $\mathcal{C}$-filtration of $M$.

A class of modules is called definable if it is closed under arbitrary direct products, direct limits, and pure submodules (cf. [12, §2.3]). Definable classes are axiomatizable by first-order formulas and they are determined by the subclass of pure-injective modules that they contain.

Let $\mathcal{C} \subseteq \operatorname{Mod} R$. Define

$$
\begin{gathered}
\mathcal{C}^{\perp_{1}}=\operatorname{Ker}_{\operatorname{Ext}_{R}^{1}}(\mathcal{C},-)=\left\{M \in \operatorname{Mod} R \mid \operatorname{Ext}_{R}^{1}(X, M)=0 \text { for all } X \in \mathcal{C}\right\} \\
{ }^{\perp_{1}} \mathcal{C}=\operatorname{Ker} \operatorname{Ext}_{R}^{1}(-, \mathcal{C}), \mathcal{C}^{\perp}=\bigcap_{i \geq 1} \operatorname{Ker} \operatorname{Ext}_{R}^{i}(\mathcal{C},-),
\end{gathered}
$$

and

$$
{ }^{\perp} \mathcal{C}=\bigcap_{i \geq 1} \operatorname{Ker} \operatorname{Ext}_{R}^{i}(-, \mathcal{C})
$$

If the class $\mathcal{C}$ has only one element, say $\mathcal{C}=\{X\}$, we write just $X^{\perp_{1}}$ instead of $\{X\}^{\perp_{1}}$, and similarly in the other cases.

A pair of classes of modules $(\mathcal{A}, \mathcal{B})$ is a cotorsion pair provided that $\mathcal{A}={ }^{\perp_{1}} \mathcal{B}$ and $\mathcal{B}=\mathcal{A}^{\perp_{1}}$. Note that for every class $\mathcal{C},{ }^{\perp} \mathcal{C}$ is a resolving class, that is, it is closed under extensions, kernels of epimorphisms and contains the projective modules. In particular, it is syzygy-closed. Dually, $\mathcal{C}^{\perp}$ is coresolving: it is closed under extensions, cokernels of monomorphisms and contains the injective modules. In particular, it is cosyzygy-closed. A pair $(\mathcal{A}, \mathcal{B})$ is called a hereditary cotorsion pair if $\mathcal{A}={ }^{\perp} \mathcal{B}$ and $\mathcal{B}=\mathcal{A}^{\perp}$. It is easy to see that $(\mathcal{A}, \mathcal{B})$ is a hereditary cotorsion pair if and only if $(\mathcal{A}, \mathcal{B})$ is a cotorsion pair and $\mathcal{A}$ is resolving if and only if $(\mathcal{A}, \mathcal{B})$ is a cotorsion pair and $\mathcal{B}$ is coresolving.

A cotorsion pair $(\mathcal{A}, \mathcal{B})$ is complete provided that every $R$-module $M$ admits a special $\mathcal{B}$-preenvelope, that is, if there exists an exact sequence of the form $0 \rightarrow$ $M \rightarrow B \rightarrow A \rightarrow 0$ with $B \in \mathcal{B}$ and $A \in \mathcal{A}$. For a class $\mathcal{C}$ of modules, the pair $\mathfrak{C}=\left({ }^{\perp}\left(\mathcal{C}^{\perp}\right), \mathcal{C}^{\perp}\right)$ is a (hereditary) cotorsion pair; it is called the cotorsion pair cogenerated by $\mathcal{C}$. Every cotorsion pair cogenerated by a set of modules is complete, [15. If all the modules in $\mathcal{C}$ have projective dimension $\leq n$, then ${ }^{\perp}\left(\mathcal{C}^{\perp}\right) \subseteq \mathcal{P}_{n}$ as well.

If $\mathcal{C}$ is a set, then a complete description of the modules in ${ }^{\perp_{1}}\left(\mathcal{C}^{\perp_{1}}\right)$ is available. In fact, by results in [15] and by [23, Theorem 22], a module belongs to ${ }^{\perp_{1}}\left(\mathcal{C}^{\perp_{1}}\right)$ if and only if it is a direct summand of a $\mathcal{C}^{\prime}$-filtered module where $\mathcal{C}^{\prime}=\mathcal{C} \cup\{R\}$. 
Clearly, ${ }^{\perp}\left(\mathcal{C}^{\perp}\right)={ }^{\perp_{1}}\left(\mathcal{C}^{\perp_{1}}\right)$ provided that a first syzygy of $M$ is contained in $\mathcal{C}$ whenever $M \in \mathcal{C}$.

A hereditary cotorsion pair $\mathfrak{C}=(\mathcal{A}, \mathcal{B})$ in $\operatorname{Mod} R$ is of countable type, and finite type, provided there is a class $\mathcal{S}$ of modules in $\mathcal{A}^{<\aleph_{1}}$, and $\mathcal{A}^{<\aleph_{0}}$, respectively, such that $\mathcal{S}$ cogenerates $\mathfrak{C}$, that is, $\mathcal{B}=\mathcal{S}^{\perp}$.

We are interested in cotorsion pairs cogenerated by an $n$-tilting module. Recall that for $n<\omega$, a module $T$ is $n$-tilting provided that

(T1) $T \in \mathcal{P}_{n}$

(T2) $\operatorname{Ext}_{R}^{i}\left(T, T^{(I)}\right)=0$ for each $i \geq 1$ and all sets $I$, and

(T3) there exist $r \geq 0$ and a long exact sequence

$$
0 \rightarrow R \rightarrow T_{0} \rightarrow \cdots \rightarrow T_{r} \rightarrow 0
$$

such that $T_{i} \in \operatorname{Add}(T)$ for each $0 \leq i \leq r$.

Here, $\operatorname{Add}(T)$ denotes the class of all direct summands of arbitrary direct sums of copies of $T$.

A class of modules $\mathcal{T}$ is $n$-tilting provided there is an $n$-tilting module $T$ such that $\mathcal{T}=T^{\perp}$. In this case, the cotorsion pair cogenerated by $T$, namely $\left({ }^{\perp} \mathcal{T}, \mathcal{T}\right)$, is called an $n$-tilting cotorsion pair. Every module in ${ }^{\perp} \mathcal{T}$ then has projective dimension $\leq n$ and the class $\mathcal{T}$ is closed under arbitrary direct sums. If $\left({ }^{\perp} \mathcal{T}, \mathcal{T}\right)$ is of countable type or of finite type, then $\mathcal{T}$ and $T$ are called $n$-tilting of countable type or of finite type, respectively.

Notice that any tilting class of finite type is definable. More generally, for any cotorsion pair $(\mathcal{A}, \mathcal{B})$ of finite type the class $\mathcal{B}$ is definable. This follows from the well-known facts that if $F$ possesses a projective resolution of finitely generated projective modules, then $\operatorname{Ext}_{R}^{n}(F,-)$ commutes with direct limits for all $n<\omega$, and that $G^{\perp_{1}}$ is closed under pure submodules whenever $G$ is finitely presented.

\section{Countably generated modules}

The aim of this section is to investigate conditions under which, for a given class $\mathcal{C}$, a module $M \in \mathcal{C}^{<\aleph_{1}}$ is a countable direct limit of objects in $\mathcal{C}^{<\aleph_{0}}$. The key idea is to look at conditions which imply that the first sygyzy module of $M$ is $\mathcal{C}^{<\aleph_{0}}$-filtered.

A particular notion of "freeness" will be used. Its origin goes back to Shelah's singular compactness theorem. The original version of the theorem appears in [21]; a shorter and more algebraic proof is given in [19]. In these papers, an algebraic structure is called "free", if it satisfies certain rather general axioms which are generalizations of properties valid for free structures and for their bases. For $R$ modules, this notion applies to more general situations than just free $R$-modules. In our setting, for instance, $M$ is considered as "free" provided that $M$ is $\mathcal{Q}$-filtered by a family $\mathcal{Q}$ of $<\mu$-presented modules for some cardinal $\mu$. Rather than stating the general axioms for the "freeness", we will concentrate on our case, where the key results concerning this notion have been collected and very well illustrated in 14 , XII.1.14] or [13].

The following result is inspired by the proof of [14, XII.1.14]. The difference in our situation is that we need to take care of possibly finitely presented modules, and to explicitly state some properties contained only implicitly in the proofs in the original papers. For the sake of further simplification, we prove the proposition 
only for $\mu=\aleph_{0}$ or $\mu=\aleph_{1}$. It could be, however, extended to any regular cardinal by following the original proofs.

Proposition 3.1. Let $\mu=\aleph_{0}$ or $\mu=\aleph_{1}$. Let $M$ be a $\mathcal{Q}$-filtered module where $\mathcal{Q}$ is a family of $<\mu$-presented modules. Then there exists a subset $\mathcal{S}$ of $\mathcal{Q}$-filtered submodules of $M$ satisfying the following properties:

(1) $0 \in \mathcal{S}$.

(2) $\mathcal{S}$ is closed under unions of arbitrary chains.

(3) For every $N \in \mathcal{S}, N$ and $M / N$ are $\mathcal{Q}$-filtered.

(4) For every subset $X \subseteq M$ of cardinality $<\mu$, there is a $<\mu$-presented module $N \in \mathcal{S}$ such that $X \subseteq N$.

Proof. Let $\left(M_{\alpha} \mid \alpha<\lambda\right)$ be a $\mathcal{Q}$-filtration of $M$ and let $\mathcal{S}$ be the set of all submodules $N$ of $M$ with the property:

$$
\text { for all } \alpha<\lambda, N \cap\left(M_{\alpha+1} \backslash M_{\alpha}\right) \neq \emptyset \text { implies } N+M_{\alpha} \supseteq M_{\alpha+1} \text {. }
$$

The properties (1) and (2) are then clear.

(3). We first show that every $N \in \mathcal{S}$ is $\mathcal{Q}$-filtered. Consider the family $\left(N \cap M_{\alpha} \mid\right.$ $\alpha<\lambda)$; then $\left(N \cap M_{\alpha+1}\right) /\left(N \cap M_{\alpha}\right)$ is zero if $N \cap\left(M_{\alpha+1} \backslash M_{\alpha}\right)=\emptyset$ and isomorphic to $M_{\alpha+1} / M_{\alpha}$ otherwise. Thus, possibly by omitting some indicies, we get a $\mathcal{Q}$ filtration of $N$.

Next, we define by induction on $\rho$ a $\mathcal{Q}$-filtration $\left(N_{\rho} / N \mid \rho<\tau\right)$ of $M / N$ such that, in addition, $N_{\rho} \in \mathcal{S}$ for all $\rho$. Let $N_{0}=N$ and assume the $N_{\rho}$ have been defined for all $\rho<\sigma(\sigma>0)$. If $\sigma$ is limit ordinal, let $N_{\sigma}=\bigcup_{\rho<\sigma} N_{\rho}$; then again $N_{\sigma} \in \mathcal{S}$ by (2). If $\sigma=\beta+1$, choose a minimal cardinal $\delta$ such that $M_{\delta} \nsubseteq N_{\beta}$. If there is no such cardinal, then $N_{\beta}=M$, and we have already the filtration. Otherwise by continuity, $\delta=\gamma+1$ for some $\gamma<\lambda$. We define $N_{\beta+1}=N_{\beta}+M_{\gamma+1}$. By (因) and by the choice of $\gamma+1$, it is easy to verify that $M_{\gamma+1} / M_{\gamma} \cong N_{\beta+1} / N_{\beta}$ via $x+M_{\gamma} \mapsto x+N_{\beta}$. So $N_{\beta+1} / N_{\beta}$ is isomorphic to an element of $\mathcal{Q}$. It remains to show that $N_{\beta+1}$ satisfies (函). Let $N_{\beta+1} \cap\left(M_{\alpha+1} \backslash M_{\alpha}\right) \neq \emptyset$, for some $\alpha<\lambda$. If $\alpha \leq \gamma$, then $N_{\beta+1}+M_{\alpha} \supseteq M_{\alpha+1}$, since $N_{\beta+1} \supseteq M_{\gamma+1} \supseteq M_{\alpha+1}$. If $\alpha>\gamma$, let $x=y+z \in N_{\beta+1} \cap\left(M_{\alpha+1} \backslash M_{\alpha}\right)$ with $y \in N_{\beta}$ and $z \in M_{\gamma+1}$. Then $y \in M_{\alpha+1}$ and $y \notin M_{\alpha}$; thus by induction $N_{\beta}+M_{\alpha} \supseteq M_{\alpha+1}$ and $N_{\beta+1}$ satisfies (函).

(4). Let $X$ be a subset of $M$ of cardinality $<\mu$. Let $\rho(X)$ be the least ordinal number such that $X \subseteq M_{\rho}$. We prove by induction on $\rho$ that there exists a $<\mu$ presented module $N \in \mathcal{S}$ such that $X \subseteq N \subseteq M_{\rho}$. If $\rho=0$ there is nothing to prove. If $\rho$ is a limit ordinal, then $X$ is not a finite subset of $M$, so $\mu=\aleph_{1}$. Let $x_{1}, x_{2}, \ldots, x_{n}, \ldots$ be an enumeration of $X$ and let $\beta_{n}<\rho$ be the least ordinal such that $\left\{x_{1}, x_{2}, \ldots, x_{n}\right\} \subseteq M_{\beta_{n}}$, for every $n<\omega$. By induction, we can define an ascending chain $\left(N_{n} \mid n<\omega\right)$ of modules of $\mathcal{S}$ with $N_{n}<\mu$-presented, $N_{n} \subseteq M_{\beta_{n}}$ and $N_{n+1} \supseteq N_{n} \cup\left\{x_{n+1}\right\}$. Set $N=\bigcup_{n<\omega} N_{n}$; by (2) $N \in \mathcal{S}$. Clearly, $X \subseteq N$ and $N$ is $<\mu$-presented contained in $M_{\rho}$.

If $\rho$ is not a limit ordinal, let $\rho=\beta+1$ for some $\beta<\lambda$. Let $L$ be a $<\mu$ generated submodule of $M_{\beta+1}$ such that $L \supseteq X$ and $L+M_{\beta}=M_{\beta+1}$. Since $M_{\beta+1} / M_{\beta}$ is $<\mu$-presented, we infer that $L \cap M_{\beta}$ is $<\mu$-generated. Let $Y$ be a generating system of $L \cap M_{\beta}$ of cardinality $<\mu$. By the induction hypothesis, there exists a $<\mu$-presented module $N_{0} \in \mathcal{S}$ such that $Y \subseteq N_{0} \subseteq M_{\beta}$. We claim that $N=L+N_{0}$ satisfies the wanted conditions. Clearly $X \subseteq N$. Moreover, $N / N_{0} \cong L /\left(N_{0} \cap L\right)$ and $L /\left(N_{0} \cap L\right) \cong M_{\beta+1} / M_{\beta}$ via $x+\left(N_{0} \cap L\right) \mapsto x+M_{\beta}$, since $N_{0} \subseteq M_{\beta}$. Thus, $N$ is $<\mu$-presented. It remains to show that $N \in \mathcal{S}$. Let 
$\gamma<\lambda$ be such that $x \in N \cap\left(M_{\gamma+1} \backslash M_{\gamma}\right)$. Then $\gamma \leq \beta$, since $N \subseteq M_{\beta+1}$. If $\gamma<\beta$, then $x \in N_{0}$, since $N_{0} \subseteq M_{\beta}$. So $N+M_{\gamma} \supseteq N_{0}+M_{\gamma} \supseteq M_{\gamma+1}$. If $\gamma=\beta$, then $N+M_{\beta}=L+M_{\beta}=M_{\beta+1}$. We conclude that $N$ satisfies the condition (図).

We will underline an immediate consequence of the condition (4) in Proposition 3.1 stating it as a corollary:

Corollary 3.2. Let $M$ be a countably generated $\mathcal{Q}$-filtered module where $\mathcal{Q}$ is a family of finitely presented modules. Then there is a filtration $\left(M_{n} \mid n<\omega\right)$ of $M$ consisting of finitely presented submodules of $M$ such that $M_{n}$ and $M / M_{n}$ are $\mathcal{Q}$-filtered for every $n<\omega$.

The following technical lemma will be of use later:

Lemma 3.3. Let $\mathcal{Q}$ be a family of finitely presented modules containing the regular module $R$. Let $M$ be a countably presented module and let

$$
0 \rightarrow K \rightarrow F \rightarrow M \rightarrow 0
$$

be a free presentation of $M$ with $F$ and $K$ countably generated. Assume that $K$ is a direct summand of a $\mathcal{Q}$-filtered module. Then, there exists an exact sequence

$$
0 \rightarrow H \rightarrow G \rightarrow M \rightarrow 0
$$

where $H$ and $G$ are countably generated $\mathcal{Q}$-filtered modules.

Proof. Let $K$ be a summand of a $\mathcal{Q}$-filtered module $P$. Since $K$ is countably generated, Proposition 3.1 implies that $K$ is contained in a countably generated $\mathcal{Q}$-filtered submodule of $P$; thus we may assume that $P$ is countably generated. By Eilenberg's trick, $K \oplus P^{(\omega)} \cong P^{(\omega)}$. Consider the exact sequence

$$
0 \rightarrow K \oplus P^{(\omega)} \rightarrow F \oplus P^{(\omega)} \rightarrow M \rightarrow 0
$$

and let $H=K \oplus P^{(\omega)} \cong P^{(\omega)}, G=F \oplus P^{(\omega)}$. Then $G$ and $H$ are countably generated $\mathcal{Q}$-filtered modules.

We apply the preceding results to the context of $n$-tilting cotorsion pairs.

Lemma 3.4. Let $(\mathcal{A}, \mathcal{B})$ be an n-tilting cotorsion pair. If $A \in \mathcal{A}$ is a countably or finitely generated module, then $A \in \mathcal{A}^{<\aleph_{1}}$ or $A \in \mathcal{A}^{<\aleph_{0}}$, respectively.

Proof. Since $\mathcal{A}$ is a resolving class, it is enough to show that every countably or finitely generated module in $\mathcal{A}$ is countably or finitely presented, respectively.

By [22, Theorems 1 and 15], $(\mathcal{A}, \mathcal{B})$ is of countable type and every module $A \in \mathcal{A}$ is $\mathcal{A}^{<\aleph_{1}}$-filtered. By Proposition 3.1 (4), a countably generated $A \in \mathcal{A}$ is countably presented.

Assume now that $A \in \mathcal{A}$ is finitely generated and let $0 \rightarrow K \rightarrow R^{n} \rightarrow A \rightarrow 0$ be a presentation of $A$. By the first part of the proof, $K$ is countably generated. Write $K=\bigcup_{n \in \omega} K_{n}$, where $K_{n}$ is a chain of finitely generated submodules of $K$. Consider the map $\phi: K \rightarrow \prod_{n<\omega} E_{n}$ defined by $\phi(x)=\left(x+K_{n}\right)_{n<\omega}$, where $E_{n}$ is an injective envelope of $K / K_{n}$ for every $n<\omega$. The image of $\phi$ is contained in $\bigoplus_{n<\omega} E_{n}$, which is an object of $\mathcal{B}$. Thus, $\phi$ extends to some $\psi: R^{n} \rightarrow \bigoplus_{n<\omega} E_{n}$. As a consequence, the image of $\phi$ is contained in $\bigoplus_{n \leq m} E_{n}$, for some $m<\omega$. Hence $K$ is finitely generated. 
In order to use an inductive argument we show now that for an $n$-tilting module $T, n \geq 1$, the cotorsion pair cogenerated by a first syzygy of $T$ is an $(n-1)$-tilting cotorsion pair.

Lemma 3.5. Let $(\mathcal{A}, \mathcal{B})$ be the cotorsion pair cogenerated by an $n$-tilting module $T$ with $n \geq 1$. Let $\left(\mathcal{A}_{1}, \mathcal{B}_{1}\right)$ be the cotorsion pair cogenerated by a first syzygy module of $T$. Then:

(1) $\left(\mathcal{A}_{1}, \mathcal{B}_{1}\right)$ is an $(n-1)$-tilting cotorsion pair,

(2) $X \in \mathcal{B}_{1}$ if and only if (any) first cosyzygy of $X$ belongs to $\mathcal{B}$,

(3) $M \in \mathcal{A}$ implies that (any) first syzygy of $M$ belongs to $\mathcal{A}_{1}$.

Proof. Let $0 \rightarrow \Omega(T) \rightarrow F \rightarrow T \rightarrow 0$ be a presentation of $T$ with $F$ free and $\Omega(T)$ a first syzygy module of $T$. Then $\Omega(T)$ has projective dimension at most $n-1$ and, by [6. Lemma 3.4], $\Omega(T)^{\perp}$ is closed under direct sums. By the characterization of tilting classes (cf. [1] or [22]), $\Omega(T)^{\perp}$ is an $(n-1)$-tilting class. Let $\left(\mathcal{A}_{1}, \mathcal{B}_{1}\right)$ be the associated cotorsion pair, namely $\mathcal{B}_{1}=\Omega(T)^{\perp}$ and $\mathcal{A}_{1}={ }^{\perp} \mathcal{B}_{1}={ }^{\perp}{ }^{\perp} \mathcal{B}_{1}$. For modules $X$ and $M$ consider exact sequences $0 \rightarrow X \rightarrow I \rightarrow \Omega^{-}(X) \rightarrow 0$ and $0 \rightarrow \Omega(M) \rightarrow F^{\prime} \rightarrow M \rightarrow 0$, where $I$ is an injective module, $F^{\prime}$ is a free module and $\Omega^{-}(X), \Omega(M)$ are first cosyzygy and syzygy modules of $X$ and $M$, respectively. Then $\operatorname{Ext}_{R}^{i}(\Omega(M), X) \cong \operatorname{Ext}_{R}^{i+1}(M, X)$ and $\operatorname{Ext}_{R}^{i+1}(M, X) \cong \operatorname{Ext}_{R}^{i}\left(M, \Omega^{-}(X)\right)$ for all $i \geq 1$. The last two statements follow immediately by these formulas.

The following provides the key ingredient for proving our main result.

Lemma 3.6. Let $(\mathcal{A}, \mathcal{B})$ be the cotorsion pair cogenerated by an $n$-tilting module $T$. Assume that the cotorsion pair $\left(\mathcal{A}_{1}, \mathcal{B}_{1}\right)$ cogenerated by a first syzygy of $T$ is of finite type. Then any countably generated module $A \in \mathcal{A}$ is isomorphic to a direct limit of a countable direct system of the form:

$$
C_{1} \stackrel{f_{1}}{\rightarrow} C_{2} \stackrel{f_{2}}{\rightarrow} C_{3} \rightarrow \cdots \rightarrow C_{n} \stackrel{f_{n}}{\rightarrow} C_{n+1} \rightarrow \cdots
$$

where the modules $C_{n}$ are in $\mathcal{A}^{<\aleph_{0}}$.

Proof. Since the cotorsion pair $\left(\mathcal{A}_{1}, \mathcal{B}_{1}\right)$ is of finite type, it is cogenerated by (a representative subset of) $\mathcal{A}_{1}^{<\aleph_{0}}$. As recalled in Section 2, $\mathcal{A}_{1}$ coincides with the class of all direct summands of the $\mathcal{A}_{1}^{<\aleph_{0}}$-filtered modules.

Fix a countably generated module $A \in \mathcal{A}$ and let

$$
0 \rightarrow K \rightarrow \bigoplus_{n<\omega} x_{n} R \rightarrow A \rightarrow 0
$$

be a presentation of $A$. Then $K \in \mathcal{A}_{1}$; thus $K$ is a summand in an $\mathcal{A}_{1}^{<\aleph_{0}}$-filtered module. By Lemma 3.4, $A$ is countably presented; so by the hypotheses and Lemma 3.3. there exists an exact sequence $0 \rightarrow H \rightarrow G \rightarrow A \rightarrow 0$, where $G$ and $H$ are countably generated $\mathcal{A}_{1}^{<\aleph_{0}}$-filtered modules. By Corollary 3.2, we can write $H=\bigcup_{n<\omega} H_{n}$ and $G=\bigcup_{n<\omega} G_{n}$ where, for every $n<\omega, H_{n}$ and $G_{n}$ are finitely presented $\mathcal{A}_{1}^{<\aleph_{0}}$-filtered modules, and $H / H_{n}, G / G_{n}$ are $\mathcal{A}_{1}^{<\aleph_{0}}$-filtered. W.l.o.g. we can assume that $H$ is a submodule of $G$. Given $n<\omega$, there is an $m(n)$ such that $H_{n} \subseteq G_{m(n)}$, and we can choose the sequence $(m(n))_{n<\omega}$ to be strictly increasing.

We claim that $G_{m(n)} / H_{n} \in \mathcal{A}^{<\aleph_{0}}$. By Lemma 3.4, it is enough to show that $G_{m(n)} / H_{n} \in \mathcal{A}$. Let $B \in \mathcal{B}$; we have to show that $\operatorname{Ext}_{R}^{1}\left(G_{m(n)} / H_{n}, B\right)=0$. But $H / H_{n}$ is $\mathcal{A}_{1}^{<\aleph_{0}}$-filtered, thus in $\mathcal{A}_{1}$, and it is immediate to check that $\mathcal{A}_{1} \subseteq$ 
$\mathcal{A}$. Moreover, $G / H \cong A \in \mathcal{A}$. Hence, every homomorphism $f: H_{n} \rightarrow B$ can be extended to a homomorphism $g: G \rightarrow B$, and the restriction of $g$ to $G_{m(n)}$ obviously induces an extension of $f$ to $G_{m(n)}$. Thus $\operatorname{Ext}_{R}^{1}\left(G_{m(n)} / H_{n}, B\right)=0$, since $G_{m(n)} \in \mathcal{A}_{1} \subseteq \mathcal{A}$.

Set $C_{n}=G_{m(n)} / H_{n}$. Since $(m(n))_{n<\omega}$ is increasing and unbounded in $\omega$, the inclusions $G_{m(n)} \subseteq G_{m(n+1)}$ induce maps $f_{n}: C_{n} \rightarrow C_{n+1}$, and $A$ is a direct limit of the direct system $\left(C_{n} ; f_{n}\right)_{n<\omega}$.

\section{Finite TYPE}

In the last step before stating the main result, we give a criterion similar to 2, Proposition 2.6] for a tilting cotorsion pair to be of finite type.

Proposition 4.1. Let $(\mathcal{A}, \mathcal{B})$ be an n-tilting cotorsion pair. Assume that every countably generated module $A \in \mathcal{A}$ is isomorphic to a direct limit of some modules in $\mathcal{A}^{<\aleph_{0}}$. Then the following are equivalent:

(1) the cotorsion pair $(\mathcal{A}, \mathcal{B})$ is of finite type;

(2) $\mathcal{B}$ is closed under pure submodules;

(3) $\mathcal{B}$ is a definable class.

Proof. The implication (3) $\Rightarrow(2)$ is clear. For the converse, recall that $\mathcal{B}$ is a coresolving class closed under direct sums. So if $\mathcal{B}$ is closed under pure submodules, then it is also closed under direct limits. $\mathcal{B}$ is always closed under products; thus (2) implies (3).

$(1) \Rightarrow(3)$ is clear.

$(3) \Rightarrow(1)$ First of all recall that, by [22, Theorem 1], every $n$-tilting cotorsion pair is of countable type. Hence $\mathcal{B}=\left(\mathcal{A}^{<\aleph_{1}}\right)^{\perp}$. Let $\mathcal{B}^{\prime}=\left(\mathcal{A}^{<\aleph_{0}}\right)^{\perp}$; then $\mathcal{B}^{\prime}$ is a definable class containing $\mathcal{B}$ and it is well known (cf. 12]) that two definable classes coincide if and only if they contain the same pure-injective modules. Let $M$ be a pureinjective module in $\mathcal{B}^{\prime}$ and let $A \in \mathcal{A}^{<\aleph_{1}}$. By hypothesis $A \cong \lim C_{n}, C_{n} \in \mathcal{A}^{<\aleph_{0}}$. Then, from a well-known result by Auslander, $\operatorname{Ext}_{R}^{i}(A, M) \cong \varliminf_{\underset{\operatorname{limt}}{i}}^{i}\left(C_{n}, M\right)=0$. Hence, $M \in \mathcal{B}$ and we conclude that $\mathcal{B}=\mathcal{B}^{\prime}$.

We are now in a position to prove our main result.

Theorem 4.2. Let $R$ be any ring and $T$ be an $n$-tilting $R$-module, $n \geq 0$. Then $T$ is of finite type.

Proof. The proof is by induction on the projective dimension $n$ of $T$.

If $n=0$, the conclusion is obvious. Next, assume that all $m$-tilting modules are of finite type for every $m<n$. Let $T$ be a tilting module of projective dimension $n$ and let $(\mathcal{A}, \mathcal{B})$ be the $n$-tilting cotorsion pair cogenerated by $T$. By 22 , Theorem $1],(\mathcal{A}, \mathcal{B})$ is of countable type; hence $\mathcal{B}=\left(\mathcal{A}^{<\aleph_{1}}\right)^{\perp}$.

Consider a free presentation of $T$ :

$$
0 \rightarrow \Omega(T) \rightarrow F \rightarrow T \rightarrow 0,
$$

and let $\left(\mathcal{A}_{1}, \mathcal{B}_{1}\right)$ be the cotorsion pair cogenerated by $\Omega(T)$. By Lemma 3.5. $\left(\mathcal{A}_{1}, \mathcal{B}_{1}\right)$ is an $(n-1)$-tilting cotosion pair; so it is of finite type by the induction hypothesis. In particular, $\mathcal{B}_{1}=\left(\mathcal{A}_{1}^{<\aleph_{0}}\right)^{\perp}$.

Let $A \in \mathcal{A}$ be a countably generated module. 
We can then apply Lemma 3.6 to conclude that there is a countable direct system of the form

$$
C_{1} \stackrel{f_{1}}{\rightarrow} C_{2} \stackrel{f_{2}}{\rightarrow} C_{3} \rightarrow \cdots \rightarrow C_{n} \stackrel{f_{n}}{\rightarrow} C_{n+1} \rightarrow \cdots
$$

where the $C_{n}$ are finitely presented modules in $\mathcal{A}$ and $A \cong \lim C_{n}$. As in [8], we consider the representation of the countable direct limit given by the exact sequence:

$$
0 \rightarrow \bigoplus_{n<\omega} C_{n} \stackrel{\phi}{\rightarrow} \bigoplus_{n<\omega} C_{n} \rightarrow \lim _{\longrightarrow} C_{n} \cong A \rightarrow 0,
$$

where, for every $n<\omega, \phi \varepsilon_{n}=\varepsilon_{n}-\varepsilon_{n+1} f_{n}$ and $\varepsilon_{n}: C_{n} \rightarrow \bigoplus_{n<\omega} C_{n}$ denotes the canonical map.

By Proposition 4.1. $(\mathcal{A}, \mathcal{B})$ is of finite type if and only if $\mathcal{B}$ is closed under pure submodules. Let $Y$ be a pure submodule of a module $Z \in \mathcal{B}$. Since $(\mathcal{A}, \mathcal{B})$ is of countable type, $Y$ is in $\mathcal{B}$ if and only if $\operatorname{Ext}_{R}^{i}(A, Y)=0$, for every $A \in \mathcal{A}^{<\aleph_{1}}$ and $i \geq 1$. Moreover, $\mathcal{A}^{<\aleph_{1}}$ is clearly closed under countable syzygies; thus $Y \in \mathcal{B}$ if and only if $\operatorname{Ext}_{R}^{1}(A, Y)=0$, for every $A \in \mathcal{A}^{<\aleph_{1}}$.

We continue now in a similar way as in the proof of $[8$, Theorem 2.5, 2.6]. We repeat the argument for the sake of completion.

Set $Z_{n}=Z$ for any $n<\omega$. Since $\mathcal{B}$ is closed under direct sums, $\bigoplus_{n<\omega} Z_{n} \in \mathcal{B}$; hence $\operatorname{Ext}_{R}^{1}\left(A, \bigoplus_{n<\omega} Z_{n}\right)=0$. From the short exact sequence (†), we see that for every homomorphism $\gamma: \bigoplus_{n<\omega} C_{n} \rightarrow \bigoplus_{n<\omega} Z_{n}$ there exists $\psi: \bigoplus_{n<\omega} C_{n} \rightarrow$ $\bigoplus_{n<\omega} Z_{n}$ such that $\psi \phi=\gamma$. By [8, Theorem 3.7] the inverse system of abelian groups

$$
\left(\operatorname{Hom}_{R}\left(C_{n}, Z\right), \operatorname{Hom}_{R}\left(f_{n}, Z\right)\right)_{n<\omega}
$$

is Mittag-Leffler. Since $Y$ is a pure submodule of $Z$ and the modules $C_{n}$ are finitely presented, [8, Theorem 4.3] yields that the inverse system

$$
\left(\operatorname{Hom}_{R}\left(C_{n}, Y\right), \operatorname{Hom}_{R}\left(f_{n}, Y\right)\right)_{n<\omega}
$$

is Mittag-Leffler, too.

Applying the functors $\operatorname{Hom}_{R}\left(C_{n},-\right)$ to the pure exact sequence

$$
0 \rightarrow Y \rightarrow Z \rightarrow Z / Y \rightarrow 0,
$$

we obtain an inverse system of pure exact sequences of the form

$$
0 \rightarrow \operatorname{Hom}_{R}\left(C_{n}, Y\right) \rightarrow \operatorname{Hom}_{R}\left(C_{n}, Z\right) \rightarrow \operatorname{Hom}_{R}\left(C_{n}, Z / Y\right) \rightarrow 0 .
$$

As $\left(\operatorname{Hom}_{R}\left(C_{n}, Y\right), \operatorname{Hom}_{R}\left(f_{n}, Y\right)\right)_{n<\omega}$ is Mittag-Leffler, we can apply 17, Proposition 13.2.2] to conclude that there is an exact sequence

$$
0 \rightarrow \lim _{\longleftarrow} \operatorname{Hom}_{R}\left(C_{n}, Y\right) \rightarrow \underset{\longleftarrow}{\lim } \operatorname{Hom}_{R}\left(C_{n}, Y\right) \rightarrow \lim _{\longleftarrow} \operatorname{Hom}_{R}\left(C_{n}, Z / Y\right) \rightarrow 0,
$$

which in turn gives the exact sequence

$$
0 \rightarrow \operatorname{Hom}_{R}(A, Y) \rightarrow \operatorname{Hom}_{R}(A, Z) \rightarrow \operatorname{Hom}_{R}(A, Z / Y) \rightarrow 0 .
$$

Therefore, we also have the exact sequence

$$
0 \rightarrow \operatorname{Ext}_{R}^{1}(A, Y) \rightarrow \operatorname{Ext}_{R}^{1}(A, Z)=0
$$

from which we deduce that $\operatorname{Ext}_{R}^{1}(A, Y)=0$ as desired. 
Remark 4.3. Let $T$ be an $n$-tilting module and $(\mathcal{A}, \mathcal{B})$ be the induced $n$-tilting cotorsion pair. Unlike in the case of countable type, it is not true in general that $T$ is $\mathcal{A}^{<\aleph_{0}}$-filtered. To see this, consider a projective module $P$ without finitely generated direct summands. Then $R \oplus P$ is a projective generator, thus a tilting module, but it is not a direct sum of finitely generated projective modules.

But we can always find an equivalent tilting module $T^{\prime}$ (that is, $T^{\perp}=\left(T^{\prime}\right)^{\perp}$ ) such that $T^{\prime}$ is $\mathcal{A}^{<\aleph_{0}}$-filtered. Indeed, let $0 \rightarrow R \rightarrow T_{0} \rightarrow X_{0} \rightarrow 0$ be a special $\mathcal{B}$-preenvelope of $R$ and $0 \rightarrow X_{i} \rightarrow T_{i+1} \rightarrow X_{i+1} \rightarrow 0$ be a special $\mathcal{B}$-preenvelope of $X_{i}$ for each $i<\omega$. Then $T^{\prime}=\left(\bigoplus_{i<n} T_{i}\right) \oplus X_{n}$ is an $n$-tilting module equivalent to $T$, 1], and we can always construct the special $\mathcal{B}$-preenvelopes so that the $X_{i}$ are $\mathcal{A}^{<\aleph_{0}}$-filtered for all $i<\omega$, [15].

\section{REFERENCES}

[1] L. Angeleri-Hügel, F. U. Coelho, Infinitely generated tilting modules of finite projective dimension, Forum Math. 13 (2001), 239-250. MR1813669 (2002b:16009)

[2] L. Angeleri-Hügel, D. Herbera, J. Trlifaj, Tilting modules and Gorenstein rings, Forum Math. 18 (2006), 211-229. MR 2218418(2007b:16014)

[3] L. Angeleri-Hügel, A. Tonolo and J. Trlifaj, Tilting preenvelopes and cotilting precovers, Algebras and Repres. Theory 4 (2001), 155-170. MR1834843(2002e:16010)

[4] M. Auslander, I. Reiten, Applications of contravariantly finite subcategories, Adv. Math. 86 (1991), 111-152. MR1097029 (92e:16009)

[5] M. Auslander, S. Smalø, Preprojective modules over Artin algebras, J. Algebra 66 (1980), 61-122. MR.591246 (83a:16039)

[6] S. Bazzoni, A characterization of $n$-cotilting and n-tilting modules, J. Alg. 273 (2004), 359372. MR2032465 (2005h:16017)

[7] S. Bazzoni, P. Eklof, J. Trlifaj, Tilting cotorsion pairs, Bull. London Math. Soc. 37 (2005), 683-696. MR2164830 (2006k:16008)

[8] S. Bazzoni, D. Herbera One dimensional tilting modules are of finite type, Algebr. Represent. Theory, in press 10.1007/s10468-007-9064-3

[9] S. Brenner, M. Butler, Generalizations of the Bernstein-Gelfand-Ponomarev reflection functors, in Proc. ICRA III LNM 832, Springer (1980), 103-169. MR607151 (83e:16031)

[10] R. R. Colby, K. R. Fuller, Tilting, cotilting and serially tilted rings, Comm. Algebra 25 (10) (1997), 3225-3237. MR1059750 (91h:16011)

[11] R. Colpi and J. Trlifaj, Tilting modules and tilting torsion theories, J. Alg. 178 (1995), 614-634. MR1359905(97e:16003)

[12] W. W. Crawley-Boevey, Infinite-dimensional modules in the representation theory of finitedimensional algebras, Algebras and modules, I (Trondheim, 1996), 29-54, CMS Conf. Proc. 23 Amer. Math. Soc., Providence, RI, 1998. MR1648602 (99m:16016)

[13] P. C. Eklof, L. Fuchs, S. Shelah, Baer modules over domains, Trans. Amer. Math. Soc. 332 (1990), 547-560. MR974514 (91c:13006)

[14] P. C. Eklof, A. H. Mekler, Almost Free Modules, 2nd Ed., North-Holland Math. Library, Elsevier, Amsterdam, 2002. MR.1914985 (2003e:20002)

[15] P. C. Eklof, J. Trlifaj, How to make Ext vanish, Bull. London Math. Soc. 33 (2001), 41-51. MR:1798574 (2001i:16015)

[16] E. Enochs, Injective and flat covers, envelopes and resolvents, Israel J. Math. 39 (1981), 33-38. MR636889 (83a:16031)

[17] A. Grothendieck, "Éléments de géométrie algébrique. III. Étude cohomologique des faisceaux cohérents. I", Inst. Hautes Études Sci. Publ. Math. 11, 1961.

[18] D. Happel, C. Ringel, Tilted algebras, Trans. Amer. Math. Soc. 215 (1976), 81-98. MR675063 (84d:16027)

[19] W. Hodges, In singular cardinality, locally free implies free, Algebra Universalis 12 (1981), 205-220. MR608664 (82i:08005)

[20] Y. Miyashita, Tilting modules of finite projective dimension, Math. Z. 193 (1986), 113-146. MR852914 (87m:16055) 
[21] S. Shelah, A compactness theorem for singular cardinals, free algebras, Whitehead problem and transversals, Israel J. Math. 21 (1975), 319-349. MR0389579 (52:10410)

[22] J. Šťovíček, J. Trlifaj, All tilting modules are of countable type, Bull. Lond. Math. Soc. 39 (2007), no. 1, 121-132.

[23] J. Trlifaj, Cotorsion theories induced by tilting and cotilting modules, Abelian Groups, Rings and Modules, Contemporary Math. 273 (2001), 285-300. MR.1817171 (2001m:16012)

[24] J. Xu, Flat covers of modules, Lecture Notes in Mathematics No. 1634, Springer-Verlag, New York (1996). MR:1438789 (98b:16003)

Dipartimento di Matematica Pura e Applicata, Universitá di Padova, Via Trieste 63, 35121 PADOVA, ITALY

E-mail address: bazzoni@math.unipd.it

Katedra algebry MFF UK, Sokolovská 83, 18675 Praha 8, Czech Republic

E-mail address: stovicek@karlin.mff.cuni.cz 